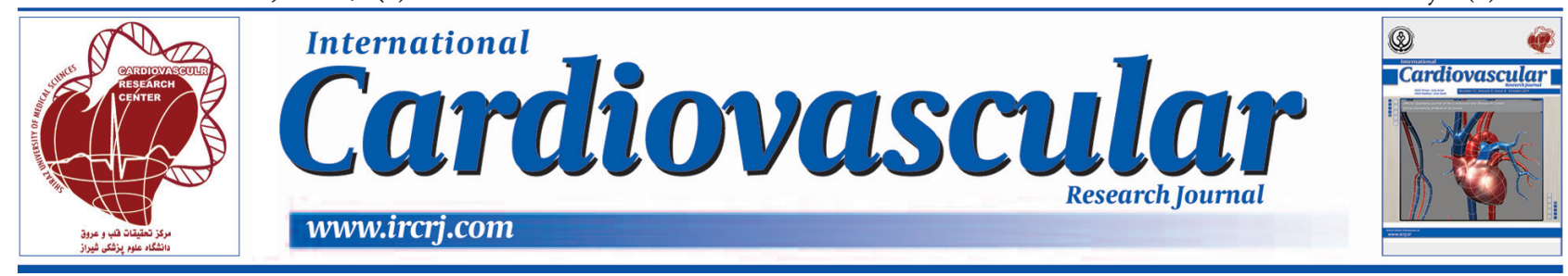

\title{
Purulent Pericarditis Caused by Streptococcus Milleri Strains; the Gained Experience from Nine Reported Cases
}

\author{
Ilias A. Kouerinis, ${ }_{1,2},{ }^{*}$ Govind Chetty, ${ }_{1}^{1}$ George Lazaros, ${ }^{2}$ Grigorios Karagkiouzis, ${ }^{2}$ Ioannis Karathanasis, ${ }^{2}$ \\ Charilaos Kantsos, ${ }^{2}$ George Georgiopoulos, ${ }^{2}$ and Graham Cooper ${ }^{1}$
}

${ }^{1}$ Department of Cardiac Surgery, Northern General Hospital, Sheffield Teaching Hospitals, Sheffield, United Kingdom

${ }^{2}$ First Department of Cardiac Surgery and Cardiology, University of Athens, Hippokration Hospital, Athens, Greece

\begin{tabular}{|c|c|}
\hline A R T I C L E I N F O & A B S T R A C T \\
\hline $\begin{array}{l}\text { Article Type: } \\
\text { Case Report }\end{array}$ & $\begin{array}{l}\text { Bacterial pericarditis caused by Streptococcus Milleri Group (SMG) strains is a } \\
\text { particularly exceptional pathology. All the eight previous reported cases were under the }\end{array}$ \\
\hline Article History: & less from this perspective. \\
\hline Received: 08 Sep 2014 & Herein, we reported a unique case of a pericardial-cutaneous fistula resulting from a \\
\hline Revised: 21 Oct 2014 & recurrent purulent pericardial effusion caused by SMG strains, which had been treated \\
\hline Accepted: 16 Dec 2014 & with open surgical drainage two months before. A thorough review of the surgical \\
\hline
\end{tabular}

Keywords:

Pericarditis

Fistula Pericardial

Surgical Complication

Antibiotics

Streptococcus Milleri

Implication for health policy/practice/research/medical education:

Purulent pericarditis is an extremely rare pathology. Only eight previous cases have been reported in the English literature and most of them were related to an underlying disease together with a variable degree of immunosuppression. The frequency, onset, treatment, complications, and recurrence rates of this infection seem to be different from other purulent pericarditis caused by other bacteria.

\section{Introduction}

Purulent pericarditis is a rare manifestation of Streptococcus Milleri Group (SMG) strain infections and most reported cases in the literature are related to an underlying disease together with a variable degree of immunosuppression (1). The frequency, onset, treatment, complications, and recurrence rates of this infection seem to be different from other purulent pericarditis caused by other bacteria.

\section{Case Presentation}

A 65-year-old patient was referred to our department for surgical drainage of his large pericardial effusion $(>3 \mathrm{~cm})$ after unsuccessful attempts for pericardiocentesis. The patient was stable and apyrexial without signs of

*Corresponding author: Ilias A. Kouerinis, NGH, Block Q5, App 9, Herries Road, S5 7AU, Sheffield, United Kingdom, Tel: 0044-7404937423,

E-mail: ikouerinis@hotmail.com tamponade. However, he complained about dizzy spells and tachycardia during the last few months. He had a long history of gingivitis and he was on prednisolone for his arthritis. His admission laboratory tests showed only an elevation of $\mathrm{WBC}=12.900 / \mathrm{mm}^{3}$.

Our initial plan for a sub-xiphoid window access failed due to multiple adhesions between the heart and the pericardium. By extending the incision and performing a standard sternotomy and pericardiotomy, a large, thick, brown pericardial effusion was revealed that was drained completely and sent for microbiology and biochemistry examination. No other intrapericardial pathology was identified.

After 48 hours, the microbiology department reported that no organisms or WBC were seen and that there was no growth of strains. Pending the final results of the cultures, the patient was discharged home on the 3rd postoperative day in excellent conditions without any antibiotics. Some days later, the final microbiology report gave the diagnosis 
of "SMG strain infection often associated with anaerobes", but the patient was lost from follow-up.

Two months later, the patient reappeared with a very high flow cutaneous fistula, stable, and without any signs of tamponade. The characteristics of this discharge were identical to those of the previous collection and the following CT scans confirmed the recurrence of the pericardial effusion and demonstrated the fistulous tract (Figure 1, 2). Nevertheless, his sternum appeared to be well healed without any signs of osteomyelitis or adhesion formation with the heart. Therefore, a subxiphoid drainage was attempted with complete success.

Postoperatively, Ceftriaxone $2 \mathrm{~g}$ and Metronidazole 400 mg were administered Intravenously (IV) for two weeks and the patient was discharged home in good condition with instructions for oral antibiotics for another 2 weeks. Two years after surgery, he remains well without any signs of recurrence.

\section{Discussion}

SMG strains (S. intermedius, S. constellatus, and $S$. anginosus) are gram-positive, non-motile, nonsporeforming, catalase-negative cocci which exhibit all 3 types of hemolysis and occur in pairs or chains. They are part of the normal flora of the mouth, nasopharynx, gastrointestinal tract, and vagina and their isolation rate is $15-45 \%$. Generally harmless, these cocci may cause several thoracic pathologies, including pulmonary

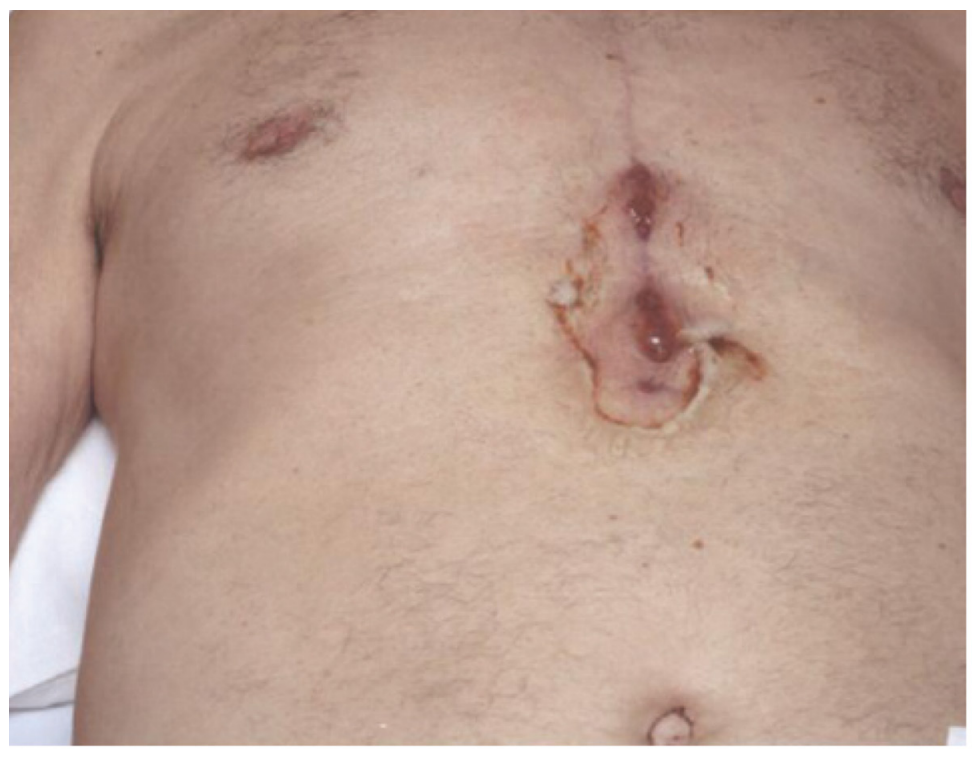

Figure 1. The Two Fistulous Tracts Draining Though His Sternal Incision

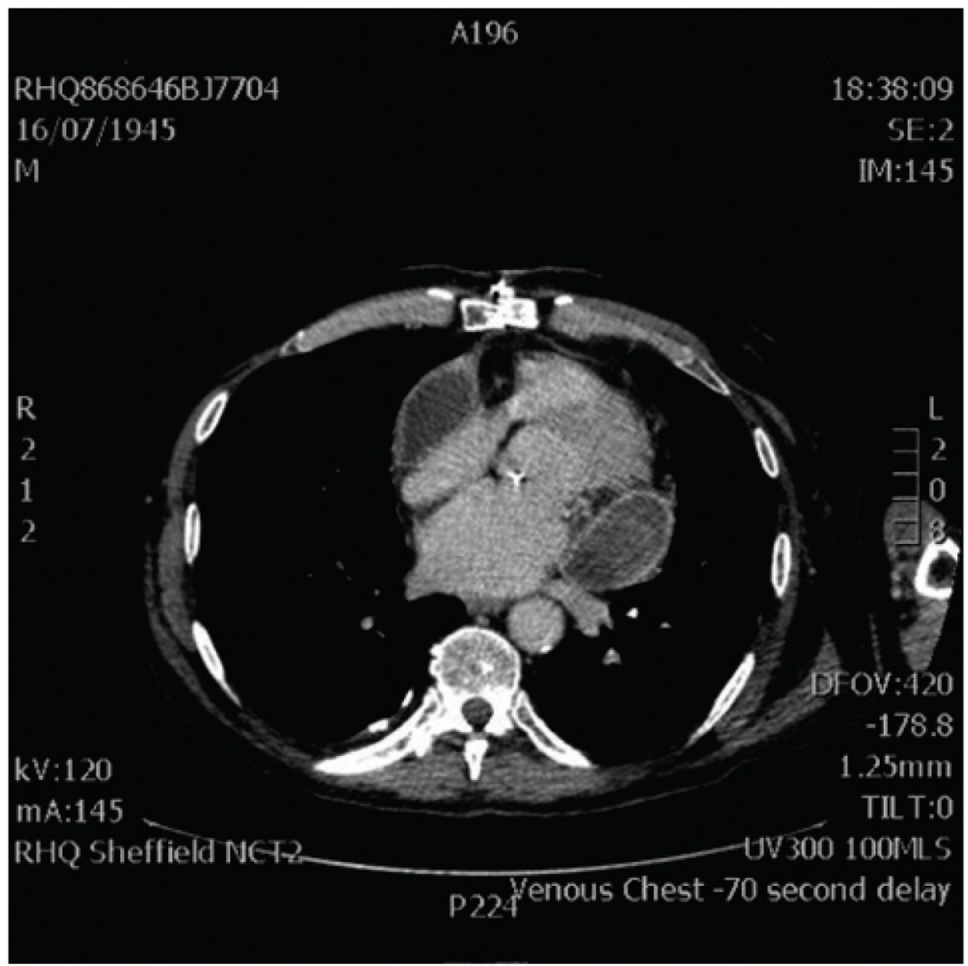

Figure 2. The CT Scans of Our Patient after the Recurrence 
infection, empyema, and purulent pericarditis, especially in immunocompromised patients. SMG strains may gain access to the thoracic space by aspiration of oral secretions, hematogenous dissemination, dissemination from contiguous foci of infection, and direct implantation during surgery or trauma. The resulting pericardial collection is thick and brown colored and emits a characteristic caramel odor when these strains are cultured in blood agar.

Purulent pericarditis caused by SMG strains is often diagnosed late in its course. In the SMG pericarditis reports, cardiac tamponade was confirmed in $33.3 \%$ of the patients on the basis of the symptoms and echocardiographic findings (Table 1). Despite that, a second episode of a life-threatening tamponade was never reported in the recurrences of SMG pericarditis following unsuccessful treatment. Pericardiocentesis under echo or fluoroscopic guidance with local anesthesia was the first treatment option in all the SMG pericarditis patients. Nevertheless, the success rate was low (44.4\%), which was attributed to the late drainage and the high viscosity of the effusion. Including our case, pericardiocentesis was not effective in the first attempt in three patients $(33.3 \%)(1,2)$ and despite the initial success in two patients $(22.2 \%)$, the catheter stopped draining soon thereafter $(3,4)$. Although there are no available data concerning the calibers of the catheters, the authors strongly suggest that the largest available caliber catheters should be used.

Following pericardiocentesis failure, pericardial drainage was attempted through a pericardial window in three cases $(1,2,4)$; one of them was later accomplished as an open procedure for pericardiotomy (2). Open drainage with sternotomy was performed as a scheduled procedure solely in our patient, while another open drainage was performed as an emergency procedure due to heart laceration during pericardiocentesis (3). The pericardium was removed in three cases based on its morphology features (2), as a prophylactic measure (3), and due to development of constrictive pathology (5).

Pericardial lavage with physiological saline at rate of 250 $\mathrm{mL} / \mathrm{hr}$ was applied in two SMG pericarditis patients $(1,2)$ as an additional measure to the tube drainage procedures.
The results of the continuous irrigation in these patients seem to be related to the duration of the treatment and the effectiveness of the drainage procedure itself. The lavage was effective when it was applied for a long period (9 days) after a successful evacuation of the sac (1). The short period of the lavage (1 day) and the incomplete drainage of the pericardial cavity resulted in a poor outcome in the other patient, which was finally treated with pericardiectomy (2).

The intrapericardial use of urokinase was performed only in one out of the nine patients (4) as a measure to unplug the malfunctioned pericardiocentesis catheter. However, it proved to be partially effective as the catheter finally blocked and the patient had to be treated with a pericardial window. A few days later, the patient developed transient constrictive pericarditis that was treated medically and eventually remitted without need for pericardiectomy. Nevertheless, the prophylactic infusion of urokinase against development of SMG constrictive pericarditis needs further investigation. Additionally, administration of corticosteroids or colchicine, as an additional measure to prevent pericardial constriction, was not applied in any of the reported cases.

Antibiotic administration in all SMG pericarditis patients was based on the sensitivity of the pericardial fluid cultures (6). The sensitivity of the pericardial fluid cultures was $100 \%$ and, thus, a pericardial tissue specimen was not necessary to set the diagnosis. SMG strains are extremely sensitive to penicillin, ampicillin, beta- lactamase-stable penicillins, and clindamycin. In the studied SMG group, only one patient had to change his antibiotic treatment from Panipenem/Betamipron to Teicoplanin due to a productive cough and the subsequent isolation of Methicillin-Resistant Staphylococcus Aureus (MRSA) from his sputum cultures (1). The suggested duration of therapy is $4-6$ weeks. Early cessation of antibiotics may result in recurrence of pericarditis and re-collection of the effusion, as in our case. When symptoms reappear within 6 weeks of the initial exacerbation despite therapy, this pericarditis should be considered as "incessant" and a more aggressive surgical approach should be planned.

Constrictive pericarditis complicated $22.2 \%(2 / 9)$ of the

\begin{tabular}{|c|c|c|c|c|c|c|}
\hline & $\begin{array}{l}\text { Tamponade }(\mathrm{T}) \\
\text { at Presentation }\end{array}$ & $\begin{array}{l}\text { Pericardio } \\
\text { Centesis }(\mathrm{P} / \mathrm{C})\end{array}$ & Result of P/C - Final Treatment & $\begin{array}{l}\text { Constrictive } \\
\text { Pathology }=>\end{array}$ & $\begin{array}{l}\text { Medical } \\
\text { Treatment }\end{array}$ & Surgical \\
\hline Kouerinis & $(-)$ & $(+)$ & $\begin{array}{l}(\mathrm{F})=>\text { Open drainage } /(\mathrm{R})=>\text { Fistula } / \\
\text { Pericardial Window }(\mathrm{PW})\end{array}$ & $(-)$ & $\mathrm{N} / \mathrm{A}$ & $\mathrm{N} / \mathrm{A}$ \\
\hline Qingbao & $(-)$ & $(+)$ & $\begin{array}{l}\text { Remaining Effusion }=>2^{\text {nd }} \mathrm{P} / \mathrm{C}, \\
\text { Heart Laceration, Open drainage }(+) \\
\text { Prophylactic Pericardiectomy }\end{array}$ & $(-)$ & N/A & $\mathrm{N} / \mathrm{A}$ \\
\hline Tokayasu & $(-)$ & $(+)$ & $\begin{array}{l}(\mathrm{F})=>\mathrm{T}(+) /(\mathrm{P} / \mathrm{W})+\text { Pericard. } \\
\text { lavage }(\mathrm{P} / \mathrm{L})\end{array}$ & $(-)$ & N/A & $\mathrm{N} / \mathrm{A}$ \\
\hline Gonzalez & $(+)$ & $(+)$ & (R)/ 1.Urokinase 2. (P/W) (late) & (+) Transient & $(+)$ & $(-)$ \\
\hline Marchal & $(-)$ & $(+)$ & Successful & $(-)$ & N/A & $\mathrm{N} / \mathrm{A}$ \\
\hline Muto (1) & $(+)$ & $(+)$ & Successful & $(-)$ & N/A & $\mathrm{N} / \mathrm{A}$ \\
\hline Muto (2) & $(-)$ & $(+)$ & Successful & $(-)$ & N/A & $\mathrm{N} / \mathrm{A}$ \\
\hline Hirata & $(+)$ & $(+)$ & Successful but constriction & $(-)$ & $(+)$ & $\begin{array}{l}(+) \\
\text { Pericardiectomy }\end{array}$ \\
\hline Akashi & $(-)$ & $(+)$ & $\begin{array}{l}(\mathrm{F})=>\mathrm{T}(+) / 1 . \mathrm{P} / \mathrm{W}+\mathrm{P} / \mathrm{L} 2 . \\
\text { Prophylactic pericardiectomy }\end{array}$ & $(-)$ & N/A & $\mathrm{N} / \mathrm{A}$ \\
\hline
\end{tabular}

Abbreviations: T, tamponade; P/C, periacardiocentesis; R, recurrence; F, failed; P/W, pericardial window; P/L, pericardial lavage. 
reported SMG pericarditis patients, which is comparable to $30 \%$ rates reported for other causes of purulent pericarditis $(5,6)$. This condition was diagnosed on the basis of the remaining tamponade physiology despite the complete drainage of the pericardial effusion and was attributed to the tendency of SMG strains to cause purulence (1). One patient showed complete resolution of the constrictive pathology without any special measures ("transient cardiac constriction") (4), while in the other, pericardiectomy had to be performed due to development of typical effusiveconstrictive pericarditis (5). When pericardiectomy is planned for constrictive pathophysiology, constrictive epicarditis (restrictive pericarditis) should have been excluded.

Prophylactic pericardiectomy to anticipate the constrictive complications was performed in two patients $(2,3)$. The decision was based on the presence of a well thickened pericardium $(20 \mathrm{~mm})$ and the strong adhesion formation. The efforts to separate the pericardium from the heart were described by the authors as vigorous (2). Only two patients $(22.2 \%)$ with SMG pericarditis had a substrate of malignancy (7). These patients had history of advanced esophageal cancer and esophago-mediastinal fistulae and both died of sepsis. Despite these poor results, the disease should be considered as curable and the survival remains $100 \%$ when no underlying malignancy is present.

\section{Acknowledgements}

The authors would like to thank Dr. Megan Carr for improving the use of English in this article.

\section{Authors' Contribution}

Ilias A. Kouerinis: study concept and design; Govid Chetty: acquisition of data; George Lazaros: drafting of the manuscript; Graham Cooper: study supervision

\section{Financial disclosure}

The authors declare that there is no conflict of interests.

\section{Funding/Support}

There is no funding/support.

\section{References}

1. Tokuyasu H, Saitoh Y, Harada T, Touge H, Kawasaki Y, Maeda $\mathrm{R}$, et al. Purulent pericarditis caused by the Streptococcus milleri group: a case report and review of the literature. Intern Med. 2009;48(12):1073-8.

2. Akashi K, Ishimaru T, Tsuda Y, Nagafuchi S, Itaya R, Hayashi J, et al. Purulent pericarditis caused by Streptococcus milleri. Arch Intern Med. 1988;148(11):2446-7.

3. Li Q, Zi J, Liu F, Li D. Purulent pericarditis caused by a bad tooth. Eur Heart J. 2013;34(11):862.

4. Salazar Gonzalez JJ, Sanchez-Rubio Lezcano J, Merchante Garcia P. [Purulent pericarditis with pneumopericardium caused by Streptococcus milleri]. Rev Esp Cardiol. 2002;55(8):861.

5. Hirata K, Asato H, Maeshiro M. A case of effusive constrictive pericarditis caused by Streptococcus milleri. Jpn Circ J. 1991;55(2):154-8.

6. Marchal LL, Detollenaere M, De Baere HJ. Streptococcus milleri, a rare cause of pericarditis; successful treatment by pericardiocentesis combined with parenteral antibiotics. Acta Clin Belg. 2000;55(4):222-4.

7. Muto M, Ohtsu A, Boku N, Tajiri H, Yoshida S. Streptococcus milleri infection and pericardial abscess associated with esophageal carcinoma: report of two cases. Hepatogastroenterology. 1999;46(27):1782-4. 